\title{
Caracterización clínica, funcional y hemodinámica de la población con hipertensión pulmonar arterial evaluada en el Instituto Nacional del Tórax
}

\author{
Mónica Zagolin B1, Eduardo Wainstein $\mathbf{G}^{2}$,
} Polentzi Uriarte $\mathbf{G}$ de $\mathbf{C}^{3}$, Claudio Parra $\mathbf{R}^{4}$.

Clinical, functional and hemodynamic features of patients with pulmonary arterial hypertension

Background: Pulmonary Arterial Hypertension is a rare, progressive and devastating disease with severe consequences in quality of life and survival. Aim: A clinical, functional and hemodynamic assessment of patients with pulmonary arterial hypertension and categorization according to severity. Material and methods: Prospective registry of patients with arterial pulmonary hypertension, hemodynamically defined. Clinical evaluation was performed using World Health Organization functional score (I to IV) and Borg dyspnea scale. Six minute walking test, echocardiography and right heart catheterization were used for functional and hemodynamic assessment. Intravenous Adenosine was used to assess vascular reactivity during the hemodynamic evaluation. Results: Twenty nine patients were included (25 women, age range 1672 years). Pulmonary hypertension was idiopathic in 11, associated to connective tissue disease in seven, associated to congenital heart disease in nine and associated to chronic thromboembolism in two. The mean lapse of symptoms before assessment was 2.9 years and $100 \%$ had dyspnea (Borg 5.1). Functional class I, II, III and IV was observed in 0, 5, 21 and 3 patients respectively. Six minutes walking test was $378 \pm 113 \mathrm{~m}$. Mean pulmonary pressure was $59.4 \pm 12.2 \mathrm{mmHg}$, cardiac index was $2.57 \pm 0.88$ and pulmonary vascular resistance index: $1798.4 \pm 855$ (dyne.sec) $/ \mathrm{cm}^{5}$. Nine patients had a mean pulmonary arterial pressure $>55 \mathrm{mmHg}$ and a cardiac index $<2.1$, considered as bad prognosis criteria. Adenosine test was positive in 17\%. Conclusions: This group of patients with Pulmonary Arterial Hypertension was mainly conformed by young females, with a moderate to severe disease (Rev Méd Chile 2006; 134: 589-95).

(Key words: Adenosine; Hemodynamic phenomena; Hypertension, pulmonary)

Recibido el 27 de mayo, 2005. Aceptado el 20 de octubre, 2005.

${ }^{1}$ Departamento de Medicina del Instituto Nacional del Tórax. ${ }^{2}$ Departamento de Medicina Interna, Clínica Las Condes, Escuela de Medicina, Universidad Andrés Bello. ${ }^{3}$ Departamento de Cardiología del Instituto Nacional de Tórax. ${ }^{4}$ Departamento de Cardiología, Hospital del Salvador.

Correspondencia a: Mónica Zagolin Blancaire. José Manuel Infante 717, Providencia, Santiago. E mail: mzagolin@torax.cl 
$\mathrm{L}$ a hipertensión pulmonar arterial (HPA) es una enfermedad infrecuente, grave, devastadora y potencialmente letal. Se ha estimado una incidencia anual, en la población general, de 1 a 2 casos por millón de habitantes ${ }^{1}$. Afecta principalmente a mujeres jóvenes que consultan ante síntomas inespecíficos, tales como disnea, fatiga o dolor torácico, que presentan 18 a 24 meses previo al diagnóstico ${ }^{2}$. El curso es progresivo y la sobrevida limitada. Se ha comunicado una sobrevida promedio de 2,8 años ${ }^{1,3}$ aunque fluctúa entre 6 y 58 meses según el nivel de gravedad y progresión de la enfermedad ${ }^{1}$.

Recientemente, la Organización Mundial de la Salud (OMS) reclasificó la HPA ${ }^{4}$ considerando las siguientes categorías: idiopática (antes denominada primaria), asociada a enfermedades del tejido conectivo (ETC), al uso de anorexígenos, HIV, shunts intracardíacos, porto-pulmonar y, además, agregó algunas entidades infrecuentes tales como: enfermedad veno-oclusiva y angiomatosis capilar pulmonar. Se consideran factores de riesgo definitivos para el desarrollo de HPA el uso o antecedente de consumo de anorexígenos tales como aminorex o fenfluramina, y el ser de sexo femenino; muy probables, el uso de anfetaminas, Ltriptofano y el embarazo e improbables, el uso de anticonceptivos, estrógenos, tabaquismo y obesi$\operatorname{dad}^{2-4}$.

La ecocardiografía es considerada el mejor método de búsqueda y detección de pacientes con HPA siendo el examen que motiva la evaluación de estos pacientes en un centro especializado ${ }^{5}$. El diagnóstico de certeza de HPA exige efectuar un cateterismo derecho para la medición directa de presiones y gasto cardíaco. Este examen, además, permite realizar la prueba de reactividad vascular, utilizando adenosina, óxido nítrico o prostaciclinas, con el propósito de reconocer a los pacientes con posibilidad de respuesta a terapia con bloqueadores del calcio ${ }^{3,6,7}$.

La evaluación clínica de estos pacientes incorpora la categorización de ellos según el nivel de clase funcional, ya sea por una variante de la NYHA propuesta por la OMS (de I a IV), que agrega a la disnea la presencia de fatiga, síncope 0 dolor torácico ${ }^{2}$.

Desde el punto de vista funcional, sin lugar a dudas, el test de caminata de 6 min es el más recomendado: de fácil realización, bajo costo $\mathrm{y}$ excelente correlación con sobrevida, progresión de la enfermedad y respuesta a la terapia ${ }^{2,3,5}$.

Son considerados criterios de mal pronóstico vital la presencia de síncopes, una capacidad funcional III o IV según la OMS, un test de caminata inferior a $332 \mathrm{~m}^{8}$, una presión de aurícula derecha (PAD) superior a $10 \mathrm{mmHg}$, una presión de arteria pulmonar media (PAPM) superior a $55 \mathrm{mmHg}$, un índice cardíaco (IC) inferior a 2,1 y una saturación de arteria pulmonar inferior a $63 \%{ }^{9}$.

En Chile se desconoce la prevalencia de la HPA, las características epidemiológicas de la población afectada, el nivel de severidad, vasorreactividad a adenosina y la evolución con o sin terapia.

El propósito de este estudio es caracterizar la población con HPA que consulta en un centro de referencia, desde un punto de vista clínico, funcional y hemodinámico y evaluar la presencia de factores pronósticos.

\section{MATERIAL Y MÉTODO}

Los pacientes referidos con diagnóstico potencial de HPA, basado en hallazgos clínicos y ecográficos fueron evaluados por un grupo multidisciplinario, conformado por un médico broncopulmonar, un reumatólogo y dos cardiólogos (hemodinamista y ecocardiografista). Fueron incluidos todos los pacientes con HPA confirmada mediante el estudio hemodinámico, que define HPA a la presencia de presión de arteria pulmonar media (PAPM) superior a $25 \mathrm{mmHg}$ en reposo o superior a $30 \mathrm{mmHg}$ en ejercicio, en ausencia de patología parenquimatosa pulmonar o cardíaca izquierda ${ }^{2-4}$. Fueron eliminados aquellos pacientes que ya estuviesen recibiendo alguna terapia específica para la HPA. La evaluación clínica se basó en la anamnesis, examen físico, puntuación de disnea de Borg de 0 a 10, capacidad funcional de la OMS (de I a IV). La escala de $\mathrm{OMS}^{2}$ clasifica a los pacientes en estadio I, II, III y IV y considera la presencia de disnea, fatiga, dolor torácico o síncopes, ya sea a esfuerzos mayores que los ordinarios, a esfuerzos habituales, menores de los rutinarios o en reposo. Se efectuaron exámenes generales de laboratorio (hemograma, perfil hepático, uricemia, pruebas tiroideas, anticuerpos antinucleares, factor reuma- 
toideo, anticardiolipinas) para objetivar el estudio etiológico. La evaluación de imágenes se basó en radiografía, tomografía axial computada con técnica helicoidal y cintigrama de perfusión pulmonar con Tecnecio marcado. Funcionalmente, fueron evaluados con el test de caminata de $6 \mathrm{~min}$, espirometría y test de difusión con monóxido de carbono. La evaluación hemodinámica no invasiva se efectuó mediante una ecotomografía de superficie realizada por operadores expertos y la invasiva, a través del cateterismo de la arteria pulmonar, efectuado siempre por el mismo equipo de trabajo. Durante el estudio hemodinámico mediante catéter de Swan Ganz en vena yugular o subclavia, se efectuaron mediciones de presiones y oximetrías a nivel de aunícula derecha, arteria pulmonar; débito cardíaco por técnica de termodilución y test de adenosina para la evaluación de reactividad vascular, este último, mediante la administración de adenosina endovenosa en infusión continua ${ }^{7}$. Se consideró un test positivo a adenosina toda disminución de al menos $20 \%$ en las cifras de PAPM o resistencia vascular pulmonar (RVP) ${ }^{6}$.

Fueron incluidos en el análisis los pacientes con HPA ya sea idiopática, asociada a ETC, VIH, drogas, cardiopatía congénita con comunicación de derecha a izquierda (Eisenmenger) y los debidos a tromboembolismo pulmonar (TEP) crónico con estudio angiográfico positivo, no susceptible de cirugía (endarterectomía). Este último grupo ha sido incluido sistemáticamente en los ensayos terapéuticos debido a que comparten elementos histopatológicos, funcionales y de respuesta farmacológica con las otras categonías de HPA.

Estadísticas. Las comparaciones entre variables de distribución no homogénea se efectuaron mediante test pareado no paramétrico de Mann-Whitney y se consideró positivo un $\mathrm{p}<0,05$.

\section{Resultados}

En el período entre junio de 2003 y marzo de 2005, 31 pacientes con diagnóstico presuntivo de HPA (clínico y ecográfico) fueron evaluados del punto de vista clínico, funcional y hemodinámico. Un paciente portador de esclerodermia, referido por disnea con estudio ecográfico, que mostraba una presión de arteria pulmonar sistólica de 65 $\mathrm{mmHg}$ resultó tener un cateterismo, tanto de reposo como post ejercicio, completamente normal, por lo que fue excluido del análisis; otro paciente, de 27 años, con diagnóstico de posible HPA idiopática, fue excluido al confirmarse el diagnóstico de pericarditis constrictiva.

La edad promedio de los 29 pacientes incorporados en el estudio fue 40,5 \pm 14 (16-72), siendo 25 de ellos mujeres. Exceptuando 6 pacientes de provincia, el resto eran todos residentes en Santiago. El tiempo de evolución de síntomas varió entre algunos meses hasta 10 años, en promedio 2,9 años. Dieciseis pacientes tenían menos de 2 años de evolución de síntomas al momento de ser evaluados. Algunos pacientes habían sido controlados en otros centros previamente, pero no se encontraban en controles regulares ni terapia específica. En la evaluación inicial, 100\% de los pacientes refirió disnea, 11 pacientes, uno o más eventos sincopales en su historia actual o pasada, 11 reportaron fatiga, 14 dolor torácico, 5 mareo y 4 la presencia de tos seca. El $83 \%$ de los pacientes tenía dos o más síntomas en el momento de la consulta al centro de referencia. En la escala funcional de la OMS, 21 pacientes se encontraban en el momento de la evaluación en capacidad funcional III y el puntaje de Borg promedio fue de 5,1 en la escala de 0 a 10.

La serie estudiada estaba constituida por los siguientes subgrupos etiológicos: 11 con HPA idiopática, 9 con Eisenmenger, 2 con TEP crónico y 7 con HPA asociada a enfermedad del tejido conectivo (ETC). De éstos últimos, 4 con lupus, 1 con enfermedad mixta del tejido conectivo (EMTC), 1 con artritis reumatoide (AR) y 1 con vasculitis. Los defectos congénitos asociados a HPA (síndrome de Eisenmenger) se debían a una comunicación interauricular tipo ostium secundum en 7 pacientes e interventricular en 2 . En la Tabla 1 se ilustra la distribución de los diferentes grupos respecto a edad, sexo y capacidad funcional.

La evaluación espirométrica resultó normal, obstructiva o restrictiva en 7, 7 y 12 pacientes, respectivamente. En los casos alterados, la limitación fue clasificada en rangos leves. El test de difusión con monóxido de carbono fue normal en 4 pacientes, levemente disminuido en 10 pacientes y moderadamente disminuido en 7 pacientes. 
Tabla 1. Características epidemiológicas de la población estudiada según etiología

\begin{tabular}{|lccccccc|}
\hline Categoría & N & Edad & Sexo F:M & II-III-IV & Borg & Distancia* & O2** \\
\hline Idiopático & 11 & 41,5 & $10: 1$ & $2-8-1$ & $6,2 \pm 2$ & $362,6 \pm 132$ & 2 \\
ETC & 7 & 39 & $6: 1$ & $2-4-1$ & $3,3 \pm 2$ & $400,7 \pm 106$ & 0 \\
Eisenmenger & 9 & 37 & $7: 2$ & $0-8-1$ & $5 \pm 2$ & $360,75 \pm 99$ & 3 \\
TEP crónico & 2 & 55,5 & $2: 0$ & $1-1-0$ & $4,5 \pm 3$ & $448 \pm 0$ & 0 \\
Total & 29 & 40,5 & $25: 4$ & $5-21-3$ & $5 \pm 2$ & $377,88 \pm 113$ & 5 \\
\hline
\end{tabular}

ETC: Enfermedad tejido conectivo. TEP: Tromboembolismo pulmonar. F:M: Relación entre sexo femenino y masculino. OMS: Clasificación funcional de la Organización Mundial de la Salud. Borg: Escala de disnea (0-10) (Promedio \pm desviación standard). *Distancia recorrida en metros promedio \pm desviación estándar. **Número de pacientes que requieren uso de oxígeno domiciliario.

No se evidenció alguna tendencia o asociación con el nivel de severidad de la HPA ni diferencias según grupo etiológico.

El examen radiológico, tomográfico y cintigráfico descartaron patología parenquimatosa pulmonar concomitante y corroboraron los hallazgos clínicos funcionales. La radiología y tomografía mostraron aumento de la arteria pulmonar y signos de hipertensión y la cintigrafía, un patrón difuso de compromiso subsegmentario bilateral. En 2 pacientes con TEP crónico, se efectuó angiografía pulmonar que descartó patología cen- tral de resorte quirúrgico en conjunto con la evaluación de cardiocirugía.

La distancia recomida promedio en el test de caminata de 6 min fue de 378 $\pm 113 \mathrm{~m}$, documentándose en 6 pacientes una distancia inferior a $332 \mathrm{~m}$

La PAP sistólica informada por ecocardiografía fue significativamente inferior a la medida mediante cateterismo $(p=0,0089)$, considerando toda la serie, aunque en el particular grupo de pacientes con ETC las cifras fueron muy similares (Tabla 2).

El estudio con adenosina fue positivo en 5 pacientes (17\%). Las variables hemodinámicas se

Tabla 2. Presión arterial sistólica medida por ecocardiografía y cateterismo derecho

\begin{tabular}{|lcc|}
\hline Etiología & PAPS-ECO & PAPS-HDN \\
\hline Idiopático & $82,3 \pm 20$ & $103,9 \pm 19$ \\
ETC & $73,7 \pm 12$ & $75,8 \pm 15$ \\
Eisenmenger & $91,2 \pm 17$ & $111,2 \pm 18$ \\
TEP & $116,5 \pm 33$ & $109 \pm 6$ \\
Total* & $85,3 \pm 20$ & $99,8 \pm 22$ \\
\hline
\end{tabular}

ETC: Enfermedad tejido conectivo. TEP: Tromboembolismo pulmonar. PAPS-ECO: Presión arterial sistólica medida por ecocardiografía en mmHg. PAPS-HDN: Presión arterial sistólica medida por cateterismo derecho. *La PAPS medida por ECO es inferior a la medida por cateterismo con un $\mathrm{p}=0,0089$ (Mann-Whitney) en todos los pacientes sin diferencias en el análisis por subgrupos. 
muestran en la Tabla 3. Diecisiete pacientes (58,6\%) tenían una PAPM superior a $55 \mathrm{mmHg}, 8$ pacientes $(27,6 \%)$ la PAD superior a $10 \mathrm{mmHg}$ y 11 pacientes, el IC inferior a 2,1. En 31\% de los pacientes se documentó la presencia de un IC inferior a 2,1 y PAPM superior a $55 \mathrm{mmHg}$, ambos criterios de mayor severidad y progresión de la enfermedad. En relación a las variables pronósticas, el grupo con HPA idiopática representa un subgrupo de mayor gravedad al compararlo con el grupo de HPA asociada a ETC $(p=0,04)$.
En la Tabla 4 se muestra la distribución de los pacientes según grupo etiológico y nivel de gravedad, considerando las variables predictivas mejor documentadas en la literatura ${ }^{3}$. El grupo con HPA idiopática presenta variables clínicas, funcionales y hemodinámicas de peor pronóstico.

Los pacientes que demostraron una respuesta positiva en el test de vasorreactividad con adenosina, iniciaron tratamiento con diltiazem y anticoagulación oral. Los pacientes sin vasorreactividad iniciaron tratamiento con sildenafil y anticoagulante.

Tabla 3. Variables hemodinámicas medidas en el cateterismo pulmonar derecho en los distintos subgrupos etiológicos

\begin{tabular}{|lccccc|}
\hline Categoría & PAD & PAPM & IC & IRVP* & SAT.AP \\
\hline Idiopático* & $9,4 \pm 8$ & $63,2 \pm 11$ & $2,24 \pm 0,63$ & $2117,5 \pm 881$ & $64,7 \pm 6,9$ \\
ETC* & $7,4 \pm 5$ & $47,71 \pm 8,3$ & $2,4 \pm 0,5$ & $1341 \pm 555$ & $68,6 \pm 11,5$ \\
Eisenmenger & $5,5 \pm 3,5$ & $64,66 \pm 12$ & $3,1 \pm 1,25$ & $1728,8 \pm 963$ & $67,7 \pm 6,3$ \\
TEP crónico & $15 \pm 13$ & $55,5 \pm 2$ & $2,3 \pm 0,3$ & $2113 \pm 0,1$ & $69,5 \pm 0,7$ \\
Total & $8,07 \pm 6,5$ & $59,4 \pm 12,2$ & $2,57 \pm 0,88$ & $1798,4 \pm 855,3$ & $67 \pm 7,7$ \\
\hline
\end{tabular}

ETC: Enfermedad tejido conectivo. TEP: Tromboembolismo pulmonar. PAD: Presión en aurícula derecha en mmHg. PAPM: Presión de arteria pulmonar media en mmHg. IC: Indice cardíaco. IRVP: Indice de resistencia vascular pulmonar dyn/segxcm-5. SAT.AP: Porcentaje de saturación arteria pulmonar. *El IRVP fue mayor en el grupo idiopático que con ETC ( $\mathrm{p}=0,04$, Mann-Whitney).

Tabla 4. D istribución de los criterios de mal pronósticos según subgrupo etiológico

\begin{tabular}{|lcccccc|}
\hline Categoría & $\begin{array}{c}\text { Distancia } \\
<332\end{array}$ & $\begin{array}{c}\text { Test } \\
\text { Adenosina } \\
(-)\end{array}$ & $\begin{array}{c}\text { PAPM } \\
>55\end{array}$ & $\begin{array}{c}\text { PAD } \\
>10\end{array}$ & $\begin{array}{c}\text { IC } \\
<2,1\end{array}$ & $\begin{array}{c}\text { SAT } \\
<63 \%\end{array}$ \\
\hline Idiopático & 3 & 8 & 9 & 4 & 6 & 4 \\
ETC & 2 & 6 & 1 & 2 & 2 & 1 \\
Eisenmenger & 2 & 8 & 6 & 1 & 2 & 3 \\
TEP crónico & 0 & 2 & 1 & 1 & 1 & 0 \\
Total & 7 & 24 & 17 & 8 & 11 & 8 \\
\hline
\end{tabular}

ETC: Enfermedad tejido conectivo. TEP: Tromboembolismo pulmonar. Distancia: En metros. PAD: Presión aurícula derecha en mmHg. PAPM: Presión de arteria pulmonar media en mmHg. IC: Indice cardíaco. SAT: Porcentaje de saturación arteria pulmonar. 
Todos ellos se encontraban en seguimiento activo con control clínico, funcional y ecocardiográfico seriados y con control hemodinámico al sexto mes.

\section{DiSCUSIÓN}

No existen trabajos nacionales que evalúen del punto de vista clínico, funcional y hemodinámico a los pacientes con HPA. Este constituye el primer informe que pretende dar a conocer algunas caractenísticas de la población nacional que se controla en un centro de referencia de patología torácica.

La serie presentada tiene algunas características similares a lo comunicado por la literatura en términos de distribución etárea, tiempo de evolución de la enfermedad y síntomas de presentación. Ha sido descrito una relación mujer:hombre de $1,7: 1^{2,3}$ y recientemente, en una serie de 162 pacientes de $3: 1^{10}$. Sin embargo, la relación en esta serie fue de 6:1, una clara preponderancia femenina que no tiene por el momento una explicación evidente. En la misma serie citada se describe una edad promedio de 42,2 años, similar a la encontrada en nuestra serie. En la población estudiada no hubo pacientes con HPA asociada a VIH, drogas anorexígenas o hipertensión portopulmonar, presumiblemente debido a que en nuestro país esos grupos son minoritarios en comparación a series europeas o norteamericanas ${ }^{3,4}$. Es también llamativa la ausencia de esclerodermia en el grupo de HPA asociada a ETC, ya que en la literatura se reportan prevalencias de HPA entre 5 y $35 \% 11,12$ lo que motivó la recomendación de la OMS de efectuar un estudio ecocardiográfico anual en este grupo de pacientes, a modo de pesquisa precoz ${ }^{2}$.

Los criterios para considerar vasorreactividad positiva son en la actualidad motivo de controversia. El estudio más extenso efectuado al respecto, usando los mismos criterios aplicados en nuestra serie $^{13}$, demostró un porcentaje de respuesta del orden de $12 \%$, algo inferior al nuestro y muy inferior a las cifras históricas de $20-30 \% 14$.

Se ha comunicado una correlación estrecha entre la distancia recorrida en el test de caminata de 6 min y el nivel de severidad y pronóstico de los pacientes con HPA, como se señala en un estudio que demuestra una menor sobrevida en los pacientes que caminaron menos de $332 \mathrm{~m}^{8}$. En nuestra serie, las distancias fueron algo superiores a dicho límite, lo que categoriza a los pacientes en un nivel de gravedad moderada en este aspecto. Del punto de vista hemodinámico, es posible considerar a esta serie en un nivel de gravedad moderado a severo, ya que un número relevante de pacientes presentó niveles de presiones en su arteria pulmonar y de índice cardíaco en los rangos catalogados de mal pronóstico. Si bien la serie es bastante pequeña para ser sometida a análisis estadístico, no existen diferencias en ninguno de los parámetros evaluados entre los diferentes grupos etiológicos, excepto en la aparente mayor edad en el grupo de TEP crónico, aunque son sólo dos pacientes y la mayor severidad en aquellos con HPA idiopática en relación a los con ETC. El resto de los grupos comparte género, edad promedio y distribución por clase y capacidad funcional.

La correlación entre las mediciones de la PAPS mediante ecocardiografía o hemodinamia fue variable, como ha sido comunicado en la literatura. Sin embargo, en la serie estudiada se observó una mejor correlación en el grupo con HPA asociada a ETC, que corresponde al grupo de menor gravedad de toda la serie. Es ampliamente reconocido el aporte de la ecocardiografía como método de pesquisa, en la selección de pacientes para el estudio hemodinámico y en el seguimiento de ellos, sin embargo, no es excluyente del estudio invasivo ${ }^{5,15}$.

Considerando principalmente las variables clínicas y hemodinámicas, la serie representa a pacientes de gravedad moderada a severa, lo que pudiera estar indicando una población genuinamente más grave 0 , con mayor probabilidad, una población con enfermedad más extensa debido a mayor tiempo de evolución libre de terapia específica. Esto último debido, quizás, a un subdiagnóstico o a la ausencia de terapias específicas disponibles con efecto en la remodelación del lecho vascular pulmonar.

En conclusión, la población de pacientes que consulta a un centro de referencia nacional concentra pacientes adultos jóvenes, de sexo femenino, principalmente con HPA idiopática, asociada a ETC o cardiopatías congénitas y con un nivel de gravedad moderado a severo. Esto último motiva la comunicación de una alerta a la comunidad en términos de la importancia de un diagnóstico y tratamiento más oportuno y a las autoridades a evaluar una mayor disponibilidad de medicamentos con acción vasodilatadora y antiproliferativa. 


\section{REFERENCIAS}

1. Rich S, Dantzker DR, Ayres SM, Bergofsky EH, Brundage BH, Detre KM et al. Primary pulmonary hypertension. A national prospective study. Ann Intern Med 1987; 107: 216-23.

2. Rich S, editor. Primary pulmonary hypertension. Executive Summary from the World Symposium. Primary Pulmonary Hypertension. World Health Organization, 1998.

3. British Cardiac Society Guidelines and Medical Practice Committee, and approved by the British Thoracic Society and the British Society of Rheumatology. Recommendations on the Management of Pulmonary Hypertension in Clinical Practice Heart 2001; Volume 86 (Supplement 1): i1-i13.

4. Simonneau G, Galué N, Rubin LJ, Langleben D, Seeger W, Dominghetti G et al. Clinical classification of pulmonary hypertension. J Am Coll Cardiol 2004; 43 (12 suppl S): 5S-12S.

5. McGoon M, Gutterman D, Steen V, Barst R, McCrory DC, Fortin TA et AL. American College of Chest Physicians. Screening, early detection, and diagnosis of pulmonary arterial hypertension. Chest 2004; 126 (1 Suppl): 14S-34S.

6. O-Sitbon O, Brenot F, Denjean A, Bergeron A, Parent F, Azarian R et al. Inhaled nitric oxide as a screening vasodilator agent in primary pulmonary hypertension. Am J Respir Crit Care Med 1995; 151 (2Pt1): 384-9.

7. Morgan JM, McCormack DG, Griffiths MJD, Morgan CJ, Barnes PJ, Evans TW. Adenosine as a vasodilator in primary pulmonary hypertension. Circulation 1991; 84: 1145-9.

8. Miramoto S, Nagaya N, Satoh T, Kyotani S, SakamaKI F, FujITA M ET AL. Clinical correlates and prognostic significance of six-minute walk test in patients with primary pulmonary hypertension. Comparison with cardiopulmonary exencise testing. Am J Respir Crit Care Med 2000; 161(2 Pt 1): 487-92.

9. D’Alonzo Ge, Barst RJ, Ayres SM, Bergofsky EH, Brundage BH, Detre KM et al. Survival in patients with primary pulmonary hypertension. Results from a national prospective registry. Ann Intern Med 1991; 115: 343-9.

10. McLaughin VV, Shimngton A, Rich S. Survival in primary pulmonary hypertension. The impact of Epoprostenol therapy. Circulation 2002; 106: 1477-82.

11. Mukerjee D, St. George D, Coleiro B, Knight C, Denton CP, Davar J et al. Prevalence and outcome in systemic sclerosis associated pulmonary arterial hypertension: application of a registry approach. Ann Rheum Dis 2003; 62: 1088-93.

12. Hachula E, Gressin V, Gullevin L, de Groote P, Cabane J, Carpentier P et al. Pulmonary arterial hypertension in systemic sclerosis: definition of a screening algothm for early detection (the ItinerAIR-Sclerodermie Study). Rev Med Interne 2004; 25: 340-7.

13. Sitbon O, Humbert M, Jaís $X$, Loos V, Hamid AM, PROVENCHER S ET AL. Long-term response to calcium channel blockers in idiopathic pulmonary arterial hypertension. Circulation 2005; 111(23): 3105-11.

14. Rich S, Kaufmann E, Levy PS. The effect of high doses of calcium-channel blockers on survival in primary pulmonary hypertension. New Engl J Med 1992; 327: 76-81.

15. Berger M, Haimowitz A, Van Tosh A, Berdoff RL, GoLDBERG E. Quantitative assessment of pulmonary hypertension in patients with tricuspid regurgitation using continuous wave Doppler ultrasound. J Am Coll Cardiol 1985; 6: 359-65.

Agradecimientos

Quisiéramos agradecer a la Srta. Mariam Torres, químico jefe de farmacia del Instituto Nacional del Tórax, por su gentil y eficiente ayuda en la disposición de adenosina para el test de reactividad vascular efectuado en los pacientes. 